Bull. Austral. Math. Soc.

$53 \mathrm{c} 30,53 \mathrm{c} 55$

VoL. 49 (1994) [239-247]

\title{
INVARIANT KÄHLER METRICS AND PROJECTIVE EMBEDDINGS OF THE FLAG MANIFOLD
}

\author{
KICHOON YANG
}

\begin{abstract}
We determine explicitly the space of invariant Hermitian and Kähler metrics on the flag manifold. In particular, we show that a Killing metric is not Kähler. The Chern forms are also computed in terms of the Maurer-Cartan form, and this calculation is used to prove that the flag manifold is projective algebraic. An explicit projective embedding of the flag manifold is also given.
\end{abstract}

\section{INTRODUCTION}

Let $G$ be a simple compact connected Lie group and also let $T$ be a maximal torus in $G$. The coset space $G / T$ is called a flag manifold. For example, taking $G=S U(n)$ and $T=S\left(U(1)^{n}\right)$ we obtain

$$
G / T=F_{1,2, \ldots, n}\left(\mathbb{C}^{n}\right)
$$

which is the space of all flags in $\mathbb{C}^{n}$. Flag manifolds are important in that they are the basic building blocks of all compact homogeneous complex spaces [3]. Moreover, a flag manifold is nonsymmetric and Kähler-Einstein of nonconstant holomorphic sectional curvature; hence, exhibits interesting differential geometric properties not encountered in, say, the complex projective space.

In this paper we take the space $F_{1,2,3}\left(\mathbb{C}^{3}\right)=S U(3) / S\left(U(1)^{3}\right)$ and determine explicitly the space of invariant Hermitian as well as Kähler metrics. In particular, we show that a Killing metric is not Kähler. We also compute the Chern forms of $F_{1,2,3}\left(\mathbb{C}^{3}\right)$ and show that the first Chern form is positive, thus establishing via the Kodaira embedding theorem that the flag manifold is projective algebraic. A noteworthy feature of our exposition is that the calculations are made quite explicitly in terms of the Maurer-Cartan form of $S U(3)$. Moreover, it will be made clear that a similar analysis applies to any flag manifold.

The second section of our paper contains a description of an embedding of $F_{1,2,3}\left(\mathbb{C}^{3}\right)$ into the complex Grassmannian $\operatorname{Gr}(3,8)$, and is based upon the work [2]. (In general, an arbitrary flag manifold of complex dimension $n$ can be embedded in a

Received 5 April 1993

Copyright Clearance Centre, Inc. Serial-fee code: 0004-9729/94 \$A2.00+0.00. 
similar way into the complex Grassmannian $\operatorname{Gr}(n, N)$, where $N$ is the real dimension of $G$ ). Via the Plücker embedding $\operatorname{Gr}(3,8) \hookrightarrow \mathbb{P}^{55}$ we can thus realise $F_{1,2,3}\left(\mathbb{C}^{3}\right)$ as a smooth projective variety. We should mention that the flag manifolds are essentially the only known Kähler-Einstein smooth projective varieties that are not of constant holomorphic sectional curvature.

\section{INVARIANT METRICS}

We consider the complex flag manifold

$$
F\left(\mathbb{C}^{3}\right)=F_{1,2,3}\left(\mathbb{C}^{3}\right)=S U(3) / S\left(U(1)^{3}\right)
$$

The group $S\left(U(1)^{3}\right)$, diagonally included in $S U(3)$, is the isotropy subgroup at the reference flag

$$
\left[\varepsilon_{1}\right] \subset\left[\varepsilon_{1} \wedge \varepsilon_{2}\right] \subset \mathbb{C}^{3},
$$

where $\left(\varepsilon_{i}\right)$ denotes the canonical basis for $\mathbb{C}^{3}$.

Let $\varepsilon_{i j}$ denote the 3 by 3 matrix with +1 at the $(i, j)$-th entry and zeros elsewhere, and put

$$
E_{i j}=\varepsilon_{i j}-\varepsilon_{j i}, \quad F_{i j}=\varepsilon_{i j}+\varepsilon_{j i} .
$$

Then the Lie algebra of $S U(3)$ decomposes as

$$
\mathfrak{s u}(3)=t \oplus \sum_{i<j} V_{i j}
$$

where $t$ denotes the Lie algebra of the maximal torus $T=S\left(U(1)^{3}\right)$, and

$$
V_{i j}=\mathbb{R} E_{i j} \oplus \sqrt{-1} \mathbb{R} F_{i j}
$$

The spaces $\left(V_{i j}\right)$ are the root spaces of $S U(3)$ with respect to $T$.

The vector subspace

$$
m=\oplus \sum_{i<j} V_{i j} \subset \mathbf{s u}(3)
$$

is an $\operatorname{Ad}(T)$-invariant complement to $t$, and it will be identified with the tangent space to $F\left(\mathbb{C}^{3}\right)$ at the identity coset via $\pi_{* e}$, where

$$
\pi: S U(3) \rightarrow F\left(\mathbb{C}^{3}\right), \quad g \mapsto g T
$$

The $s u(3)$-valued Maurer-Cartan form $\Omega=\left(\Omega_{j}^{i}\right)_{1 \leqslant i, j \leqslant 3}$ decomposes into

$$
\Omega=\Omega_{t} \oplus \sum_{i<j} \Omega_{V_{i j}}
$$


where $\Omega_{V_{i j}}$ denotes the $V_{i j}$-component of $\Omega$. So

$$
\Omega_{V_{i j}}=\operatorname{Re} \Omega_{j}^{i} \otimes E_{i j}+\operatorname{Im} \Omega_{j}^{i} \otimes F_{i j} .
$$

The standard complex structure of $F\left(\mathbb{C}^{3}\right)$ is given by letting the pullbacks of the following complex-valued 1 -forms to be of type $(1,0)$ :

$$
\Omega_{2}^{1}, \Omega_{3}^{1}, \Omega_{3}^{2} .
$$

Note that the real and imaginary parts of these forms constitute the $m$-component of the Maurer-Cartan form. By way of notation we put

$$
\omega_{j}^{i}=s^{*} \Omega_{j}^{i},
$$

where $s$ is a local section of the principal fibration $S U(3) \rightarrow F\left(\mathbb{C}^{3}\right)$.

THEOREM. Any invariant Hermitian metric on $F\left(\mathbb{C}^{3}\right)$ is given by the $\operatorname{Ad}(T)$ invariant tensor product

$$
d s_{(a, b, c)}^{2}=a^{2} \omega_{2}^{1} \otimes \bar{\omega}_{2}^{1}+b^{2} \omega_{3}^{1} \otimes \bar{\omega}_{3}^{1}+c^{2} \omega_{3}^{2} \otimes \bar{\omega}_{3}^{2},
$$

where $a, b, c$ are positive constants. Thus the totality of invariant Hermitian metrics on $F\left(\mathbb{C}^{3}\right)$ is naturally parameterised by $\left(\mathbb{R}^{+}\right)^{3}$.

The above result is a straightforward consequence of the following rather general consideration. Let $G$ be a simple compact connected Lie group, and consider the root space decomposition with respect to a maximal torus $T$

$$
\mathfrak{g}=t \oplus \sum_{i=1}^{n} V_{i}
$$

Recall that any two bi-invariant metrics on $G$ are constant multiples of each other; hence, the root spaces of a simple Lie group are defined canonically. The subspace $\mathfrak{m}=\oplus \sum_{i=1}^{n} V_{i}$ is identified with the tangent space $T_{T}(G / T)$; an invariant metric on $G / T$ corresponds, via restriction, to an $\operatorname{Ad}(T)$-invariant inner product in $\mathrm{m}$. Now $\operatorname{Ad}(T)$ restricted to each $V_{i}$ is irredicible, and consequently each $V_{i}$ possesses only a one-dimensional family of $\operatorname{Ad}(T)$-invariant inner products. Thus the totality of $\operatorname{Ad}(T)$ invariant inner products in $\mathfrak{m}$ is given by

$$
\left\{\left.\sum c_{i} \cdot \kappa\right|_{v_{i}}, c_{i}<0\right\} \cong\left(\mathbb{R}^{+}\right)^{n},
$$

where $\kappa$ denotes the Killing form of $G$. 
THEOREM. The metric $d s_{(a, b, c)}^{2}$ on $F\left(\mathbb{C}^{3}\right)$ is Kähler if and only if

$$
(a, b, c)=\lambda(1, \sqrt{2}, 1)
$$

for some $\lambda \in \mathbb{R}^{+}$.

Proof: A unitary coframe for the metric $d s_{(a, b, c)}^{2}$ is given by

$$
\theta^{1}=a \omega_{2}^{1}, \quad \theta^{2}=b \omega_{3}^{1}, \quad \theta^{3}=c \omega_{3}^{2}
$$

We then have

$$
d \theta^{i}=-\psi_{j}^{i} \wedge \theta^{j}+\tau^{i}
$$

where $\left(\psi_{j}^{i}\right)$ is the $u(3)$-valued connection form and $\left(\tau^{i}\right)$ are the torsion forms. It is well-known that the metric $d s_{(a, b, c)}^{2}$ is Kähler if and only if the torsion forms vanish identically. Using the Maurer-Cartan structure equations of $S U(3)$ we calculate that

$$
d\left[\begin{array}{l}
\theta^{1} \\
\theta^{2} \\
\theta^{3}
\end{array}\right]=\left[\begin{array}{ccc}
\omega_{1}^{1}-\omega_{2}^{2} & \frac{a}{b c} \bar{\theta}^{3} & 0 \\
\frac{-b}{2 a c} \theta^{3} & \omega_{1}^{1}-\omega_{3}^{3} & \frac{b}{2 a c} \theta^{1} \\
0 & \frac{-c}{a b} \bar{\theta}^{1} & \omega_{2}^{2}-\omega_{3}^{3}
\end{array}\right] \wedge\left[\begin{array}{l}
\theta^{1} \\
\theta^{2} \\
\theta^{3}
\end{array}\right] .
$$

It follows that $\left(\tau^{i}\right) \equiv 0$ if and only if

$$
\frac{a}{b c}=\frac{b}{2 a c} \text { and } \quad \frac{b}{2 a c}=\frac{c}{a b} .
$$

And this is so if and only if $(a, b, c)=\lambda(1, \sqrt{2}, 1)$ for some $\lambda>0$.

DEFINITION: The metric $d s_{(1, \sqrt{2}, 1)}^{2}$ will be called the normal Kähler metric.

REMARK. In general, on a flag manifold $G / T$ of complex dimension $n$ the space of invariant Hermitian metrics is parameterised by $\left(\mathbb{R}^{+}\right)^{n}$. And amongst these exactly $\mathbb{R}^{+}$many of them are Kähler. More precisely, any two invariant Kähler metrics on $G / T$ are homothetically equivalent to each other.

A Killing metric on $F\left(\mathbb{C}^{3}\right)$ is, by definition, a Hermitian metric coming from a negative multiple of the Killing form restricted to $\mathrm{m}$. Since for any $X, Y$ in the Lie algebra $\operatorname{su}(n)$

$$
\operatorname{trace}(\operatorname{ad} X \circ \operatorname{ad} Y)=c \cdot(\operatorname{trace}(X \cdot Y))
$$

for some dimensional constant $c$, we see that a Killing metric is given by

$$
d s_{\lambda(1,1,1)}^{2}, \quad \lambda \in \mathbb{R}^{+} .
$$

This observation combined with the above theorem yields the following somewhat surprising corollary. 
COROLlaRY. A Killing metric on $F\left(\mathbb{C}^{3}\right)$ is not Kahler.

We now compute the Chern forms of $F\left(\mathbb{C}^{3}\right)$ using the normal Kähler metric $d s_{(1, \sqrt{2}, 1)}^{2}$. From the computation above we see that the connection matrix of this metric with respect to the unitary coframe

$$
\theta^{1}=\omega_{2}^{1}, \quad \theta^{2}=\sqrt{2} \omega_{3}^{1}, \quad \theta^{3}=\omega_{3}^{2}
$$

is given by

$$
\left(\psi_{j}^{i}\right)=\left[\begin{array}{ccc}
\omega_{1}^{1}-\omega_{2}^{2} & \left(1 / \sqrt{2} \bar{\theta}^{3}\right. & 0 \\
(-1 / \sqrt{2}) \theta^{3} & \omega_{1}^{1}-\omega_{3}^{3} & (1 / \sqrt{2}) \theta^{1} \\
0 & (-1 / \sqrt{2}) \bar{\theta}^{1} & \omega_{2}^{2}-\omega_{3}^{3}
\end{array}\right] .
$$

The curvature forms $\chi=\left(\chi_{j}^{i}\right)$ are computed from the formulae

$$
\chi_{j}^{i}=d \psi_{j}^{i}+\psi_{k}^{i} \wedge \psi_{j}^{k}
$$

We calculate that

$$
\begin{gathered}
\chi_{1}^{1}=2 \theta^{1} \wedge \bar{\theta}^{1}+\frac{1}{2} \theta^{2} \wedge \bar{\theta}^{2}-\frac{1}{2} \theta^{3} \wedge \bar{\theta}^{3}, \\
\chi_{2}^{2}=\frac{1}{2} \theta^{1} \wedge \bar{\theta}^{1}+\theta^{2} \wedge \bar{\theta}^{2}+\frac{1}{2} \theta^{3} \wedge \bar{\theta}^{3}, \\
\chi_{3}^{3}=-\frac{1}{2} \theta^{1} \wedge \bar{\theta}^{1}+\frac{1}{2} \theta^{2} \wedge \bar{\theta}^{2}+2 \theta^{3} \wedge \bar{\theta}^{3}, \\
\chi_{2}^{1}=\frac{1}{2} \theta^{1} \wedge \bar{\theta}^{2}, \quad \chi_{3}^{1}=-\frac{1}{2} \theta^{1} \wedge \bar{\theta}^{3}, \quad \chi_{3}^{2}=\frac{1}{2} \theta^{2} \wedge \bar{\theta}^{3}, \\
\chi_{j}^{i}=-\bar{\chi}_{i}^{j} .
\end{gathered}
$$

Let $c_{k}(\chi), 1 \leqslant k \leqslant 3$, denote the $k$-th Chern form of $F\left(\mathbb{C}^{3}\right)$ constructed using $\chi$ so that

$$
\begin{gathered}
c_{1}(\chi)=\frac{i}{2 \pi} \operatorname{trace} \chi, \\
c_{2}(\chi)=\left(\frac{i}{2 \pi}\right)^{2} \sum_{i<j}\left(\chi_{i}^{i} \wedge \chi_{j}^{j}-\chi_{j}^{i} \wedge \chi_{i}^{j}\right), \\
c_{3}(\chi)=\left(\frac{i}{2 \pi}\right)^{3} \operatorname{det} \chi .
\end{gathered}
$$

We find that

(2) $c_{2}(\chi)=\left(-3 / 4 \pi^{2}\right)\left(\theta^{1} \wedge \bar{\theta}^{1} \wedge \theta^{2} \wedge \bar{\theta}^{2}+2 \theta^{1} \wedge \bar{\theta}^{1} \wedge \theta^{3} \wedge \bar{\theta}^{3}+\theta^{2} \wedge \bar{\theta}^{2} \wedge \theta^{3} \wedge \bar{\theta}^{3}\right)$,

$$
c_{3}(\chi)=\left(-3 i / 4 \pi^{3}\right)\left(\theta^{1} \wedge \bar{\theta}^{1} \wedge \theta^{2} \wedge \bar{\theta}^{2} \wedge \theta^{3} \wedge \bar{\theta}^{3}\right) \text {. }
$$


Theorem. The flag manifold $F\left(\mathbb{C}^{3}\right)$ equipped with the normal Kähler metric is Kähler-Einstein with constant scalar curvature 24.

Proof: The Kähler form of $\left(F\left(\mathbb{C}^{3}\right), d s_{(1, \sqrt{2}, 1)}^{2}\right)$ is given by

$$
\Lambda=\frac{i}{2} \sum \theta^{i} \wedge \bar{\theta}^{i}
$$

Then from (1) we see that

$$
c_{1}(\chi)=\frac{2}{\pi} \Lambda
$$

showing that $F\left(\mathbb{C}^{3}\right)$ is Kähler-Einstein. Now the scalar curvature $s$ satisfies

$$
c_{1}(\chi)=(s / 12 \pi) \Lambda
$$

and $s=24$.

Incidentally, the Kähler manifold $\left(F\left(\mathbb{C}^{3}\right), d s_{(1, \sqrt{2}, 1)}^{2}\right)$ is not of constant holomorphic sectional curvature. To see this recall that the curvature forms $\left(\chi_{\beta}^{\alpha}\right)$, written relative to a unitary coframe $\left(\theta^{\alpha}\right)$, of a Kähler manifold with constant holomorphic sectional curvature $c$ are given by

$$
\chi_{\beta}^{\alpha}=\frac{c}{4}\left(\theta^{\alpha} \wedge \bar{\theta}^{\beta}+\delta_{\beta}^{\alpha} \sum \theta^{\gamma} \wedge \bar{\theta}^{\gamma}\right)
$$

ThEOREM. The flag manifold $F\left(\mathbb{C}^{3}\right)$ is projective algebraic.

Proof: The formula (1) shows that the first Chern class of $F\left(\mathbb{C}^{3}\right)$ is positive. Then the anticanonical line bundle $K^{*} \rightarrow F\left(\mathbb{C}^{3}\right)$ must be ample since

$$
c_{1}\left(F\left(\mathbb{C}^{3}\right)\right)=c_{1}\left(\Lambda^{3}\left(F\left(\mathbb{C}^{3}\right)\right)\right)=c_{1}\left(K^{*}\right)
$$

Thus by the Kodaira embedding theorem a suitable pluri-anticanonical linear system (that is, the linear system of divisors associated with a large enough positive integral power of $K^{*}$ ) gives rise to a projective embedding of $F\left(\mathbb{C}^{3}\right)$.

REMARK. A similar consideration shows that any flag manifold is projective algebraic. In fact, [2] shows that a flag manifold is a rational variety.

\section{The flag manifold as a subVariety of the complex Grassmannian}

Let $G$ be a semisimple simply connected and connected compact Lie group, and fix a maximal torus $T \subset G$. Then there is a unique holomorphic Lie group $G^{C}$ with Lie algebra $\mathfrak{g}^{C}$ (the complexification of $\mathfrak{g}$ ) containing $G$. 
REMARK. We are making the simple connectivity assumption here merely to avoid certain technical complications. After all, if $\widetilde{G}$ is the quotient of $G$ by any finite invariant subgroup and $\widetilde{T}$ is the image of $T$ under the projection $G \rightarrow \widetilde{G}$, then the spaces $G / T$ and $\tilde{G} / \widetilde{T}$ are well-known to be diffeomorphic.

A root of the holomorphic Lie group $G^{C}$ is an element $\alpha$ of $\left(t^{C}\right)^{*}$ such that the root space

$$
\mathfrak{g}_{\alpha}=\left\{v \in \mathfrak{g}^{C}: \operatorname{ad}_{h}(v)=[h, v]=\alpha(h)(v), h \in \mathfrak{t}^{C}\right\}
$$

is nontrivial. The set of all roots of $G^{C}$ will be denoted by $\Delta \subset\left(t^{C}\right)^{*}$. We then have the root space decomposition

$$
\mathfrak{g}^{C}=\mathfrak{t}^{C} \oplus \sum_{\boldsymbol{\alpha} \in \Delta} \mathfrak{g}_{\boldsymbol{\alpha}}
$$

We fix a system of positive roots in $\Delta$, and write

$$
\Delta=\Delta_{+} \cup \Delta_{-}
$$

We then put

$$
\mathfrak{b}=\mathfrak{t}^{C} \oplus \sum_{\alpha \in \Delta^{+}} \mathfrak{g}_{\alpha}, \quad \mathfrak{n}=\oplus \sum_{\alpha \in \Delta^{-}} \mathfrak{g}_{\alpha} .
$$

The algebra $\mathfrak{b}$ is a Borel subalgebra and $\mathfrak{n}$ is nilpotent. We let $B$ (respectively, $N$ ) denote the analytic subgroup of $\mathfrak{b}$ (respectively, $\mathfrak{n}$ ) in $G^{C}$. It can then be verified that

$$
G \cap B=T, \quad G \cap N=\{e\},
$$

implying that the map

$$
G / T \rightarrow G^{C} / B, \quad g T \mapsto g B
$$

is a diffeomorphism.

We are interested in the case

$$
\begin{gathered}
G=S U(3), \quad T=S\left(U(1)^{3}\right), \\
G^{C}=S L(3, \mathbb{C}), \quad B=\text { upper triangular matrices } .
\end{gathered}
$$

We shall identify the complex Lie algebra $\mathfrak{s}(3, \mathbb{C})$ with $\mathbb{C}^{8}$ via the map

$$
e_{a} \mapsto \varepsilon_{a}, \quad 1 \leqslant a \leqslant 8
$$

where

$$
\begin{aligned}
& e_{1}=\varepsilon_{13}, \quad e_{2}=\varepsilon_{12}, \quad e_{3}=\varepsilon_{23}, \\
& e_{4}=\varepsilon_{11}-\varepsilon_{22}, \quad e_{5}=\varepsilon_{22}-\varepsilon_{33}, \\
& e_{0}=\varepsilon_{32}, \quad \varepsilon_{7}=\varepsilon_{21}, \quad \varepsilon_{8}=\varepsilon_{13} .
\end{aligned}
$$


Note that

$$
\mathfrak{t}^{C}=\operatorname{span}\left\{e_{4}, e_{5}\right\}
$$

In addition, the roots of $S L(3, \mathbb{C})$ corresponding to the root vectors $e_{1}, e_{2}$ and $e_{3}$ form a system of positive roots $\Delta_{+}$so that

$$
\begin{aligned}
& \mathfrak{b}=\mathfrak{t}^{C} \oplus \mathbb{C} e_{1} \oplus \mathbb{C} e_{2} \oplus \mathbb{C} e_{3}, \\
& \mathbf{n}=\mathbb{C e}_{6} \oplus \mathbb{C}_{7} \oplus \mathbb{C}_{8} .
\end{aligned}
$$

From the formula

$$
\left[\varepsilon_{i j}, \varepsilon_{k l}\right]=\delta_{j k} \varepsilon_{i l}-\delta_{l i} \varepsilon_{k j}
$$

we compute the image of the adjoint map ad: $\mathfrak{s l}(3, \mathbb{C}) \rightarrow \mathfrak{g l}(k, \mathbb{C})$, where $\mathfrak{g l}(8, \mathbb{C})$ is the set of all 8 by 8 complex matrices. Write

$$
\operatorname{ad}(\mathfrak{s l}(3, \mathbb{C}))=\left\{X=\sum_{a=1}^{8} x_{a} \operatorname{ad}\left(e_{a}\right): x_{a} \in \mathbb{C}\right\}
$$

Calculations show that $X$ is given by

$(*)$

$$
\left[\begin{array}{cccccccc}
x_{4}+x_{5} & -x_{3} & x_{2} & -x_{1} & -x_{1} & 0 & 0 & 0 \\
-x_{6} & 2 x_{4}-x_{5} & 0 & -2 x_{2} & x_{2} & x_{1} & 0 & 0 \\
x_{7} & 0 & -x_{4}+2 x_{5} & x_{3} & -2 x_{3} & 0 & -x_{1} & 0 \\
-x_{8} & -x_{7} & 0 & 0 & 0 & 0 & x_{2} & x_{1} \\
-x_{8} & 0 & -x_{6} & 0 & 0 & x_{3} & 0 & x_{1} \\
0 & x_{8} & 0 & x_{6} & 2 x_{6} & x_{4}-2 x_{5} & 0 & -x_{2} \\
0 & 0 & -x_{8} & 2 x_{7} & -x_{7} & 0 & -2 x_{4}+x_{5} & x_{3} \\
0 & 0 & 0 & x_{8} & x_{8} & -x_{7} & x_{6} & -x_{4}-x_{5}
\end{array}\right] .
$$

And ad (b) consists of those matrices with $x_{6}=x_{7}=x_{8}=0$.

Let $G L(8, \mathbb{C})$ act on the complex Grassmannian $\operatorname{Gr}(3,8)$ in the usual manner, and also let $K$ denote the isotropy subgroup at the 3-plane $\left[\varepsilon_{1} \wedge \varepsilon_{2} \wedge \varepsilon_{3}\right]$. Thus

$$
K=\left\{\left[\begin{array}{ll}
A & * \\
0 & *
\end{array}\right] \in G L(8, \mathbb{C}): A \in G L(3, \mathbb{C})\right\}
$$

The Lie algebra of $K$ is given by

$$
\mathfrak{f}=\left\{\left[\begin{array}{ll}
X & * \\
0 & *
\end{array}\right] \in \mathfrak{g l}(8, \mathbb{C}): X \in \mathfrak{g l}(3, \mathbb{C})\right\} .
$$

From (*) we then observe that

$$
\operatorname{ad}(\mathfrak{b})=\mathfrak{f} \cap \operatorname{ad}(\mathfrak{s l}(3, \mathbb{C})) .
$$


Let $G_{1} \subset G L(8, \mathbb{C})$ denote the group generated by ad $(\mathfrak{s l}(3, \mathbb{C}))$, and let $B_{1} \subset$ $G L(8, \mathbb{C})$ denote the group generated by ad $(\mathfrak{b})$. Then $G_{1}$ is locally isomorphic to $G^{C}$ and $B_{1}$ is locally isomorphic to $B$; in such a case it is well-known (see [1], for example) that the spaces $G_{1} / B_{1}$ and $G^{C} / B$ are biholomorphically identified with each other. Moreover, $(\dagger)$ shows that the map

$$
\Phi: G_{1} / B_{1} \rightarrow G L(8) / K, \quad g B_{1} \mapsto g K
$$

is a well-defined monomorphism. We have thus arrived at the following theorem.

THEOREM. The flag manifold $F\left(\mathbb{C}^{3}\right)=G_{1} / B_{1}$ is a smooth subvariety of the complex Grassmannian $\mathrm{Gr}(3,8)$ via the map $\Phi$.

It would be quite interesting to relate the projective embedding

$$
G_{1} / B_{1} \subset \mathrm{Gr}(3,8) \subset \mathbb{P}^{55}
$$

to a pluri-anticanonical projective embedding of $F\left(\mathbb{C}^{3}\right)$, whose existence we established earlier.

\section{REFERENCES}

[1] A. Borel, 'Sur la cohomologie des espaces fibrés principaux et des espaces homogènes de groupes de Lie compacts', Ann. of Math. 57 (1953), 115-207.

[2] M. Goto, 'On algebraic homogeneous spaces', Amer. J. Math. 76 (1954), 811-313.

[3] H.C. Wang, 'Closed manifolds with homogeneous complex structure', Amer. J. Math. 76 (1954), 1-32.

Department of Mathematics

Arkansas State University

State University, AR 72467

United States of America 